\title{
Pulmonary Distribution of Lucinactant and Poractant Alfa and Their Peridosing Hemodynamic Effects in a Preterm Lamb Model of Respiratory Distress Syndrome
}

MICHAEL H. TERRY, T. A. MERRITT, BENJAMIN HARDING, HOBE SCHROEDER, JEANETTE MERRILL-HENRY, JAN MAZELA, TIMOTHY J. GREGORY, ROBERT SEGAL, GORDON G. POWER, AND ARLIN B. BLOOD

Department of Respiratory Care [M.H.T., J.M.-H.]; Department of Pediatrics [T.A.M., B.H., A.B.B.]; Center for Perinatal Biology [H.S., G.G.P., A.B.B.], Loma Linda University School of Medicine, Loma Linda, California 92354; Discovery Laboratories, Inc. [J.M., T.J.G., R.S.], Warrington, Pennsylvania 18976; Department of Neonatology [J.M.], Poznan University of Medical Sciences, 60-535 Poznan, Poland

\begin{abstract}
Tracheal instillation of surfactant to premature newborns improves their survivability but may transiently obstruct airways resulting in undesirable acute effects on cerebral blood flow $(\mathrm{CBF})$ and oxygenation. The acute peridosing hemodynamic effects of surfactant administration may be avoided by minimizing the volume of surfactant administered, but smaller surfactant volumes may also result in less even distribution of surfactant throughout the lung. These experiments were undertaken to compare responses to two surfactants with different dose volumes (porcine-derived poractant alfa, $2.5 \mathrm{~mL} / \mathrm{kg}$ vs peptide-based synthetic lucinactant, 5.8 $\mathrm{mL} / \mathrm{kg}$ ) given to newly delivered lambs at $85 \%$ gestation. Both surfactants resulted in similar improvements in blood gas values, a doubling of dynamic compliance, increases in brain tissue oxygen tension, and stable blood pressure with no significant change in CBF. Distribution of surfactant throughout the lungs was more uniform with lucinactant than poractant alfa when assessed by labeled microspheres. We conclude that improvements in lung mechanics, gas exchange, and changes in CBF are comparable for a porcine-derived and peptide-containing synthetic surfactant, despite instilled volumes differing by 2-fold. Intrapulmonary distribution of surfactant is more uniform after a larger volume is instilled. (Pediatr Res 68: 193-198, 2010)
\end{abstract}

$\mathrm{T}$ he use of exogenous pulmonary surfactants in neonatal medicine has greatly improved the survival of premature infants. However, the administration of surfactant, which is performed by liquid bolus instillation into the endotracheal tube, can result in transient airway obstruction and has been associated with rapid changes in oxygen saturation, heart rate, systemic blood pressure, and cerebral blood flow (CBF) (Table S1, http://links.lww.com/PDR/A60). Concern exists that such peridosing effects of surfactant administration may predispose the fragile premature cerebral vasculature to hemorrhage, an injury that is common in this patient population (1),

Received February 3, 2010; accepted April 28, 2010.

Correspondence: Arlin B. Blood, Ph.D., 11175 Campus Street, 11121C Coleman, Loma Linda University, Loma Linda, CA 92354; e-mail: ablood@llu.edu

Supported by an unrestricted grant from Discovery Laboratories, Inc.

Authors from Discovery Laboratories, Inc. [R.S., J.M., T.G.] had input into protocol design but not in data collection nor in analyses and had only minor editorial contribution to the preparation of the article.

Supplemental digital content is available for this article. Direct URL citations appear in the printed text and are provided in the HTML and PDF versions of this article on the journal's Web site (www.pedresearch.org). and may be associated with long-term neurologic sequelae. The adverse peridosing effects of surfactant administration may potentially be reduced by decreasing the rate or volume of surfactant instillation to avoid airway obstruction. However, evidence suggests that decreasing the rate (2) or volume (3) of surfactant bolus instillation results in more uneven distribution of surfactant within the lung.

Factors influencing the pulmonary distribution of exogenously administered surfactant include viscosity, density, gravity, injection rate, orientation of the trachea with respect to gravity, tracheal diameter, surface tension, respiratory rate, positive airway pressure, and catheter size for injection. It has been reported that uniformity of surfactant distribution improves as the volume of surfactant administered is increased $(3,4)$. Therefore, although minimizing the volume of instillate may be desirable with regard to avoiding peridosing adverse events, even distribution of the surfactant to the distal airways may be favored by rapid administration of a large volume.

There also may be biochemical responses (e.g. vasoactivity and inflammatory response) to bioactive substances in animalderived surfactant. This raises the possibility that peridosing responses to animal-derived and synthetically produced surfactant products may differ apart from volume or method of administration.

In this study, we measured the effects of surfactant administration on $\mathrm{CBF}$, brain tissue oxygen tensions $\left(\mathrm{tPO}_{2}\right)$, arterial blood pressure, and heart rate in an established premature lamb model of neonatal respiratory distress syndrome. We compared a porcine-derived surfactant (poractant alfa) administered at $2.5 \mathrm{~mL} / \mathrm{kg}$ with a peptide-containing, synthetically produced surfactant (lucinactant) administered at $5.8 \mathrm{~mL} / \mathrm{kg}$. Our null hypothesis was that no detectable differences would exist between peridosing responses to either of the surfactant. We also tested whether the intrapulmonary distribution of the two surfactants would differ using labeled microspheres admixed with the surfactants.

Abbreviations: CBF, cerebral blood flow; LDF, laser Doppler flowmetry; tPO2, brain tissue oxygen tension 


\section{METHODS}

Surgical instrumentation. Animal care protocols were preapproved by the Loma Linda University Institutional Animal Care and Use Committee. Pregnant ewes were obtained from Nebeker Ranch (Lancaster, CA) at 124 to $126 \mathrm{~d}$ gestation (term $\sim 147 \mathrm{~d}$ ). At 48 and $24 \mathrm{~h}$ before delivery of the lambs, the ewes were treated with $12 \mathrm{mg}$ of betamethasone intramuscularly to enhance lung maturity, a procedure chosen to mimic obstetrical management of pregnant women with anticipated preterm delivery.

The ewes were given $500 \mathrm{mg}$ of thiopental intravenously (i.v.) and then intubated and ventilated with $2 \%$ isoflurane. The fetal head was exteriorized through a midline incision in the abdomen of the ewe. A 4.0 endotracheal tube was inserted $7 \mathrm{~cm}$ through a tracheotomy at the second tracheal ring, locating the tip $\sim 1 \mathrm{~cm}$ above the carina. The endotracheal tube was clamped on insertion to prevent loss of lung fluid. Each lamb was instrumented bilaterally with a composite laser Doppler flowmetry (LDF) and $\mathrm{tPO}_{2}$ probe (diameter $\sim 400 \mu \mathrm{m}$; Oxyford Optronix, Ltd, Oxford, UK) inserted through a burr hole drilled on either side of the skull, $\sim 1 \mathrm{~cm}$ posterior to the coronal suture and $0.5 \mathrm{~cm}$ lateral to the sagittal suture as previously described (5). The tip of the probe was advanced into the parietal cortex $\sim 0.5 \mathrm{~cm}$ below the dura, a position previously determined to position the probe tip in the gray matter (5). After surgical instrumentation, the lamb was delivered and weighed, the umbilical cord ligated and severed, amniotic fluid was suctioned out of the airway using a $15-\mathrm{cm}$ cathether, and mechanical ventilation was initiated. Catheters were inserted into an umbilical artery and vein for the measurement of arterial blood gases, blood pressure, and administration of medications. The lambs received an i.v. infusion of $5 \%$ glucose in water at $6 \mathrm{~mL} / \mathrm{h}, 1 \mathrm{mg} / \mathrm{kg} / \mathrm{h}$ of ketamine, and $0.1 \mathrm{mg} / \mathrm{kg} / \mathrm{h}$ of pancuronium throughout the experiment.

Ventilation. Mechanical ventilation was initiated at 60 breaths per minute, peak inspiratory pressure of $25-\mathrm{cm} \mathrm{H}_{2} \mathrm{O}$, positive end-expiratory pressure (PEEP) of 5-cm $\mathrm{H}_{2} \mathrm{O}$, an inspiratory/expiratory ratio of $1: 2$, and an $\mathrm{FiO}_{2}$ of 1.0. Ventilator settings were adjusted to maintain an arterial $\mathrm{Po}_{2}>90$ torr, $\mathrm{PCO}_{2}$ of 40 to 60 torr, and $\mathrm{pH}$ of 7.25 to 7.5. Trometamol [Tris(hydroxymethyl) aminomethane buffer (THAM)] was administered i.v. to treat acidosis as needed.

Protocol timeline. A total of 12 premature lambs were studied, six of which received poractant alfa and six received lucinactant. Surfactant was administered by bolus into the endotracheal tube after a 20 -min period of baseline measurements. The lambs were monitored for a total of $180 \mathrm{~min}$ from the time of initiating surfactant administration.

To assess the ability of the cerebral vasculature to autoregulate flow in this premature animal model, the effect of arterial hypotension on CBF was examined in six lambs ( $n=3$ per group) just before euthanization. Data from all six animals were pooled based on the assumption that the type of surfactant administered did not affect autoregulatory capacity. Sixty to $100 \mathrm{~mL}$ of arterial blood was withdrawn during a period of $\sim 5 \mathrm{~min}$ to achieve blood pressures ranging from baseline to close to $20 \mathrm{~mm} \mathrm{Hg}$. The data were analyzed by linear regression of arterial blood pressure and CBF.

Surfactant administration. Poractant alfa (Curosurf ${ }^{\circledR}$; Dey Laboratories, Napa, CA) was administered in adherence to its package insert. Vials were first warmed to room temperature and then the appropriate dose (based on 2.5 $\mathrm{mL} / \mathrm{kg}$ or $200 \mathrm{mg} / \mathrm{kg}$ ) was drawn into a $5-\mathrm{mL}$ syringe. With the lamb in prone position and tilted $45^{\circ}$ to achieve right side gravity dependence, half of the surfactant volume was instilled over $\sim 15 \mathrm{~s}$. This was done using a 5-French catheter cut to a length even with the distal opening of the endotracheal tube, through a $\mathrm{NEO}_{2}$-Safe adapter (B\&B Medical Technologies, Carlsbad, CA) without disruption of PEEP. The lamb was ventilated for $1 \mathrm{~min}$. The lamb was then repositioned left side down, and an identical dose of surfactant was administered, followed again by $1 \mathrm{~min}$ of ventilation. The lambs were not suctioned for at least $1 \mathrm{~h}$ after the surfactant administration. The head of the bed was elevated $\sim 15^{\circ}$ to minimize regurgitation of the surfactant into the ventilator circuit.

Lucinactant $\left(\right.$ Surfaxin $\left.^{\circledR}\right)$ was provided by Discovery Laboratories Inc. (Warrington, PA) and administered as divided bolus doses. Vials were warmed to $44^{\circ} \mathrm{C}$ in a dry block heater for $15 \mathrm{~min}$ before administration. Lucinactant was drawn into a $10-\mathrm{mL}$ syringe (based on $5.8 \mathrm{~mL} / \mathrm{kg}, 175$ $\mathrm{mg} / \mathrm{kg}$ ) and administered in 4 quarter doses without interruption of the ventilatory circuit using a $\mathrm{NEO}_{2}$-Safe adapter. The lambs were positioned right side down for the first and third doses and left side down for the second and fourth doses. The lambs were not suctioned for $1 \mathrm{~h}$ after the surfactant administration, and again the head of the bed was elevated $15^{\circ}$ to prevent significant regurgitation of the surfactant into the ventilator circuit.

Microspheres. To assess the uniformity of surfactant distribution within the lungs, labeled microspheres were admixed into the surfactant administered to five lambs in each study group. The microspheres (BioPAL, Inc., Worcester, MA) of 1 to $2 \mu \mathrm{m}$ diameter ( $\sim 30$ million spheres with $7.2 \mathrm{mg}$ of stable isotope metal) were provided in water suspension. The spheres were centri-
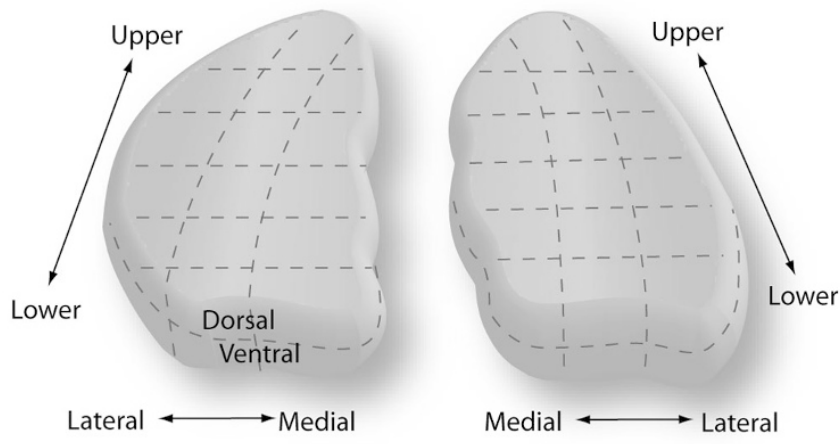

Figure 1. Lung sectioning for evaluation of microsphere/surfactant distribution.

fuged at 10,000 $\mathrm{g}$ for $1 \mathrm{~min}$, the supernatant was removed, and the pellet then suspended and mixed thoroughly in the surfactant solution immediately before the administration as previously described (6)

On euthanization of the lamb, the lungs were removed from the chest en bloc, placed in supine position on a flat surface, and frozen at $-70^{\circ} \mathrm{C}$. The frozen lungs were weighed and then sectioned into a total of 72 pieces, as shown in Figure 1. Each piece was weighed immediately to minimize errors because of evaporative loss

The microspheres were quantified by neutron radioactivation of the metals followed by gamma counter analysis, resulting in a measurement of dpm (BioPAL Inc., Worcester, MA) in pieces of known weight and location.

Blood pressure, heart rate, and pulmonary compliance. Arterial blood pressure was sampled at $200 \mathrm{~Hz}$ throughout the experiments using a pressure transducer (Cobe, Lakewood, CO), converted to a digital signal (Powerlab, ADInstruments, CO Springs, CO), and recorded by computer (Chart v5.2 for Macintosh, ADInstruments). Heart rate was calculated from the arterial blood pressure wave form.

Airway pressure and flow were monitored continuously via a variable orifice pneumotachometer (Bicore I, Cardinal Health). Dynamic compliance $\left(\mathrm{mL} / \mathrm{cm} \mathrm{H}_{2} \mathrm{O}\right)$ and exhaled tidal volume $(\mathrm{ml} / \mathrm{kg})$ were recorded at 20 and 5 min before surfactant administration and every 15 min thereafter.

Blood gases. Arterial blood gases ( $0.4 \mathrm{~mL}$ blood sample) including $\mathrm{Po}_{2}$, $\mathrm{PCO}_{2}, \mathrm{pH}$, base excess (ABL-5, Radiometer, Copenhagen Denmark), oxyhemoglobin saturation, $\mathrm{O}_{2}$ content, and methemoglobin saturation (OSM3, Radiometer) were measured at $-20,-15,-5,5,10,20$, and 30 min relative to surfactant administration and then in 15-min intervals relative to the initiation of surfactant throughout the remainder of the experiment.

CBF and Oxygen Tension. CBF was sampled at $100 \mathrm{~Hz}$ via LDF (OxyFlo; Oxford Optronix, Oxford, UK). Because LDF provides relative changes in flow, values were expressed as a percent of mean baseline flow during the 20-min period immediately preceding surfactant administration. Cerebral $\mathrm{tPO}_{2}$ was recorded at $10 \mathrm{~Hz}$ (OxyLite; Oxford Optronix). The OxyLite probe provides an absolute measure of tissue oxygenation by ruthenium dye fluorescence.

Data analysis. To assess rapid changes after surfactant administration, the continuously recorded parameters $\left(\mathrm{CBF}, \mathrm{tPO}_{2}\right.$, blood pressure, and heart rate) were re-sampled into 10 -s averages from 5 min prior until $15 \mathrm{~min}$ after initiation of surfactant administration. The continuously recorded data were also resampled into 1-min averages from $20 \mathrm{~min}$ prior until $180 \mathrm{~min}$ after the initiation of surfactant administration. Significance of changes in parameters with time was assessed by one-way ANOVA with repeated measures (Graphpad Prizm v5.0 for Macintosh). If the one-way ANOVA reached significance, a Bonferroni adjustment was used to determine which time points were measurably different from baseline. For blood gas, compliance, and tidal volume, measurements at $-5 \mathrm{~min}$ were used as a baseline. Significant differences between the lucinactant and poractant alfa groups were detected using two-way ANOVA.

Surfactant distribution in each tissue sample was normalized to tissue weight such that relative distribution was expressed as follows:

$$
\frac{\mathrm{dpm} \text { in tissue sample/dpm in whole lung }}{\text { sample weight/total lung weight }}
$$

These results were expressed as logarithms (base 10) to achieve a normal distribution. In this manner, a perfectly homogenous microsphere distribution on a per gram tissue basis would have resulted in a value of $0\left(\log _{10}\right.$ of 1$)$ for 


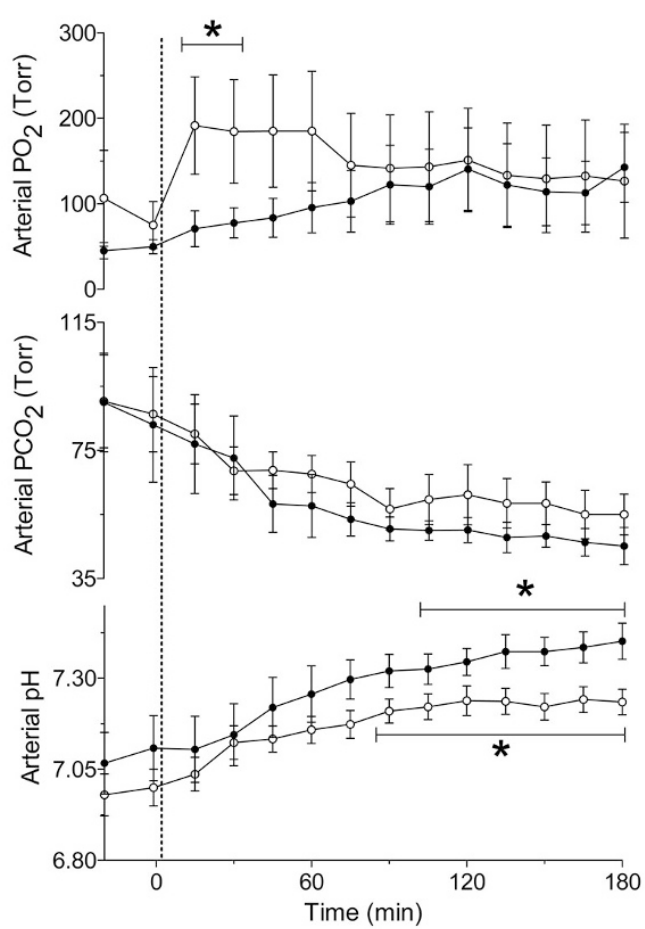

Figure 2. Three-hour time course of blood gas responses after tracheal instillation of poractant alfa $(\bigcirc)$ and lucinactant $(\bullet)$ given to newly delivered fetal sheep at $85 \%$ gestation. Note improving metabolic and respiratory acidosis for both surfactants. *Denotes statistical difference from baseline $(p<0.01)$ for arterial $\mathrm{Po}_{2}$ (poractant alfa only) and $\mathrm{pH}$ (both lucinactant and poractant alfa).

all pieces. Data were evaluated to quantify differences in the microsphere distribution between the six upper to lower divisions, the three medial to lateral divisions, and the two ventral (dependent) to dorsal (nondependent) divisions. These assessments were made for both groups using one-way ANOVA for the upper-to-lower and medial-to-lateral divisions, and a $t$ test for the dependent to nondependent divisions. If a significant difference was found with ANOVA, Bonferroni's and Dunnett's adjustments were performed to identify differences between specific tissue sections. To demonstrate overall homogeneity between the two study groups, the distribution data are presented both as a histogram as well as fit to a Gaussian curve (Graphpad Prism v5.0 for Macintosh, Graphpad Software, LA Jolla, CA).

\section{RESULTS}

Twelve lambs were studied, six with poractant alfa ( 3 males and 3 females) and six with lucinactant ( 2 males and 4 females). The mean weight \pm SEM of poractant alfa animals was $2.6 \pm 0.1 \mathrm{~kg}$, and the mean weight of lucinactant animals was $2.8 \pm 0.1 \mathrm{~kg}$.

Blood gases. All lambs developed respiratory and metabolic acidosis, which was treated by intravascular volume expansion with $10 \mathrm{~mL} / \mathrm{kg}$ normal saline and boluses of THAM at $3 \mathrm{~mL} / \mathrm{kg}$ for a base excess $<-7 \mathrm{mM}$. After surfactant instillation, the respiratory acidosis improved for lambs in both study groups (Fig. 2). Arterial $\mathrm{PCO}_{2}$ decreased and $\mathrm{pH}$ increased significantly during the course of the experiment $(p<0.01)$. Arterial $\mathrm{Po}_{2}$ tended to increase in both groups, rising significantly after treatment with poractant alfa, with considerable variation among lambs. The difference between groups did not reach statistical significance.

Pulmonary mechanics. Mean airway pressures (data not shown) tended to decrease during the course of the experiment,

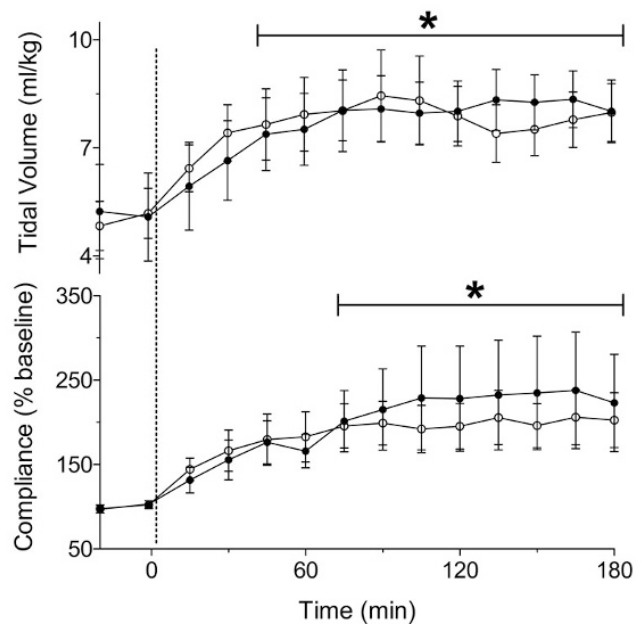

Figure 3. Time course of respiratory parameters after instillation of poractant alfa $(\bigcirc)$ and lucinactant $(\bigcirc)$ into newly delivered premature lambs. Note 2-fold increase in dynamic compliance for both surfactants. *Statistical difference from baseline $(p<0.01)$.

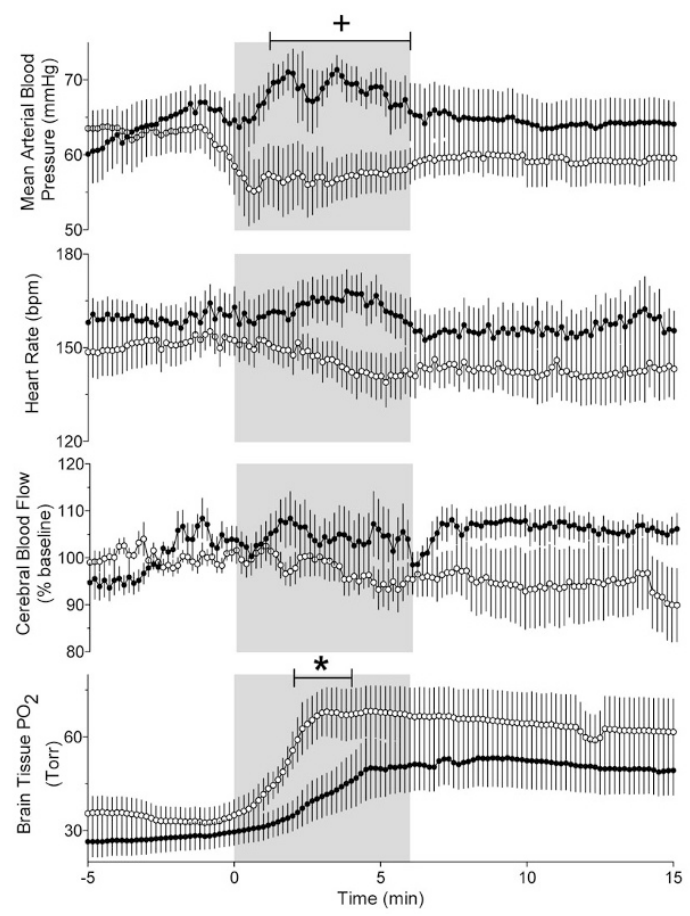

Figure 4. Cardiovascular responses after instillation of poractant alfa $(\bigcirc)$ and lucinactant $(\mathbf{O})$. "+" denotes significant difference between arterial blood pressures of the two groups $(p<0.05)$. *Denotes more rapid rise in $\mathrm{tPO}_{2}$ after poractant alfa $v s$ lucinactant $(p<0.01)$.

but changes were not statistically significant with respect to time or treatment. Within 75 min after surfactant instillation both tidal volume and dynamic compliance had increased (Fig. 3). During the first $90 \mathrm{~min}$ of the experiment, compliance increased to a plateau of $\sim 200 \%$ of baseline values, a significant increase for both lucinactant and poractant alfa $(p<0.05)$.

Arterial blood pressure, $C B F$, and brain tissue oxygenation. Mean pressures fell in the poractant alfa group and rose in the lucinactant group such that the two groups were transiently significantly different from one another shortly after surfactant administration (Fig. 4) but not throughout the re- 


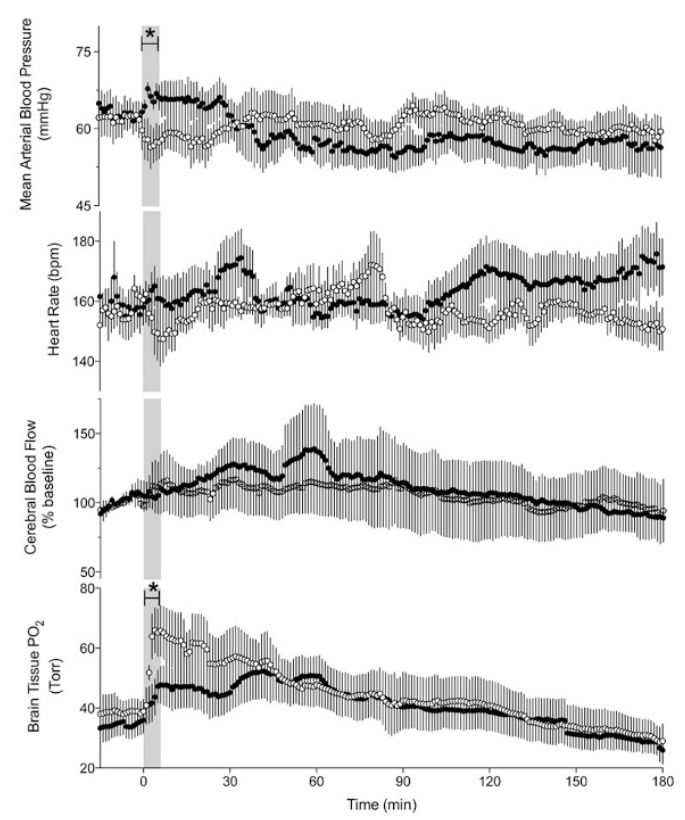

Figure 5. Cardiovascular responses during 3-h observation period. Note cortical blood flow does not change for either surfactant, and $\mathrm{tPO}_{2}$ returns to values measured before surfactant administration. *Denotes significant difference between poractant alfa and lucinactant $(p<0.01)$.

mainder of the experiment. However, CBF did not change significantly after surfactant administration in either of the two treatment groups (Fig. 4). Administration of poractant alfa resulted in a statistically significant increase in $\mathrm{tPO}_{2}(p<$ 0.01). $\mathrm{tPO}_{2}$ increased more rapidly in animals treated with poractant alfa compared with lucinactant, and remained significantly higher for $\sim 2 \min (p<0.05)$. Apart from changes in arterial blood pressure and $\mathrm{tPO}_{2}$ as noted above, there were no significant differences between the two groups nor with respect to baseline values for blood pressure, heart rate, $\mathrm{CBF}$, or $\mathrm{tPO}_{2}$ during the $3 \mathrm{~h}$ after surfactant administration (Fig. 5).

The observation that CBF was not affected by fluctuations in arterial blood pressure during surfactant administration may indicate active autoregulation of flow by the cerebral vasculature. In response to gradual withdrawal of blood to lower blood pressure, $\mathrm{CBF}$ remained unchanged until mean arterial pressures fell below $\sim 35 \mathrm{~mm} \mathrm{Hg}$. This result is consistent with autoregulation of $\mathrm{CBF}$ within the range of blood pressures observed during surfactant administration (Fig. 6).

Surfactant distribution. The distribution data comprise results of 360 samples taken from five lungs in each group. The average weight of individual tissue samples was $0.95 \pm 0.04 \mathrm{~g}$ in the animals given lucinactant and $0.89 \pm 0.03$ in animals given poractant alfa. An average of 24,323 \pm 1712 microspheres and 20,797 \pm 2956 microspheres were counted in the individual pieces of lucinactant and poractant lungs, respectively, a number of spheres that enables surfactant distribution measurements with $>3 \%$ precision $95 \%$ of the time (7). Less lucinactant was distributed to the lowest segment of the lung when compared with the upper segments $(p<0.01)$, and less poractant alfa was distributed to the uppermost and lowest segment of the lung when compared with the middle segments $(p<0.001)$. No significant differences were found in surfac-

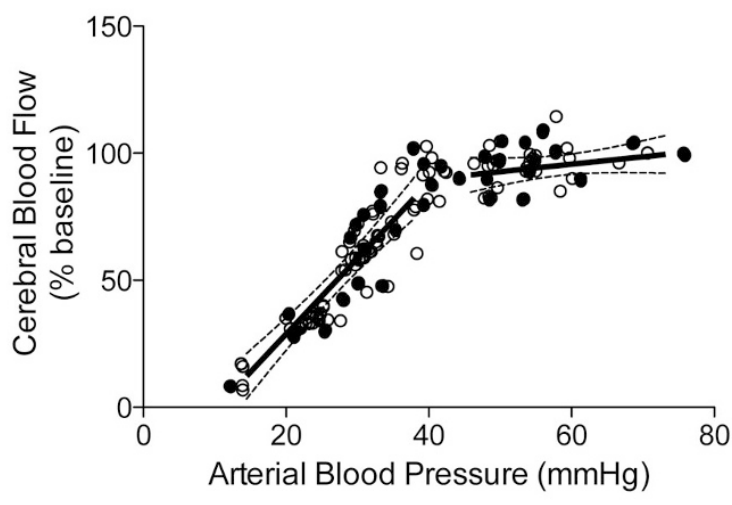

Figure 6. Changes in $\mathrm{CBF}$ in response to changes in arterial pressure. Results are from six lambs (lucinactant lambs $=\bigcirc$, poractant alfa lambs $=\mathbf{0}$ ) at $\sim 125 \mathrm{~d}$ gestation. Note the pattern characteristic of autoregulation, with little change in blood flow until arterial pressure falls below $\sim 40 \mathrm{~mm} \mathrm{Hg}$. Linear regressions (solid lines) of data $<40 \mathrm{~mm} \mathrm{Hg}$ revealed a significant effect of arterial blood pressure on CBF (slope $=3.0 \pm 0.2, p<0.001$ ), while there was no effect $>40 \mathrm{~mm} \mathrm{Hg}$ (slope $=0.3 \pm 0.1, p>0.1$ ).

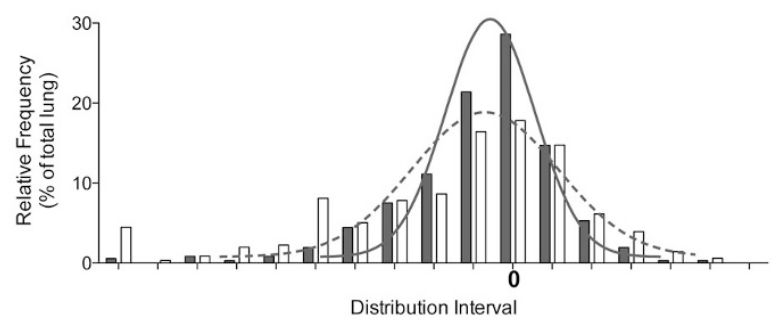

Figure 7. Percentage of lung tissue containing different relative amounts of microspheres mixed with instilled surfactant. The distribution following lucinactant ( $\square$, solid line) instillation is more uniform than following poractant alfa ( $\square$, dashed line, $p<0.01$ ), as demonstrated by the tighter distribution around 0 , the value of ideal distribution.

tant distribution from the medial to lateral portions of the lung for either lucinactant or poractant alfa. Nor were significant differences observed between dependent and nondependent lung segments for either surfactant treatment (Fig. S2, http://links.lww.com/PDR/A60). The relative surfactant distribution per gram of lung tissue in lambs receiving lucinactant was clustered more tightly around 0 (the value expected if distribution were homogenous) compared with poractant alfa, as demonstrated by the width (kurtosis) of the Gaussian curve (Fig. 7, lucinactant $=3.27$, poractant alfa $=0.709 ; p<0.01$ ).

\section{DISCUSSION}

In these experiments, we observed no significant effect of surfactant administration on CBF, despite the volume dose of lucinactant being twice as large as poractant alfa. The administration of poractant alfa resulted in a rapid increase in brain tissue $\mathrm{PO}_{2}$ and lower arterial blood pressures when compared with lucinactant. The distribution of lucinactant in the lung was significantly more uniform than poractant alfa, but both surfactants were not evenly distributed to the upper and lower portions of the lung.

Peridosing effects of surfactant administration. The goals of surfactant therapy are to improve pulmonary mechanical 
function and gas exchange and to decrease pulmonary morbidities. For infants at high risk for respiratory distress syndrome or with established respiratory disease, surfactants are given by a variety of bolus injection techniques that include variable positioning of the chest, instillation in half or quarter dosing schemes, and differences in ventilatory support during the dosing procedures. Dosing with surfactants and associated manipulation of the infant can result in cyanosis, bradycardia, and reflux of surfactant into the endotracheal tube (8), raising concerns regarding potential adverse effects on the cardiovascular homeostasis, particularly in the fragile cerebrovasculature. There have been numerous studies examining the effects of surfactant instillation on arterial blood pressure and cerebral hemodynamics, with mixed results reported. In a total of 13 studies, using ultrasound Doppler flowmetry, xenon clearance, or electromagnetic flowmetry to measure the effect of surfactant administration on $\mathrm{CBF}$, nine studies reported significant decrease in $\mathrm{CBF}$, three studies reported no effect on $\mathrm{CBF}$, and three studies reported an increase in CBF (Table S1, http://links.lww.com/PDR/A60). In six studies using near infrared resonance spectrometry to measure changes in cerebral blood volume, two studies reported increases suggestive of increased blood flow and four studies reported no changes. The reported effects of surfactant administration on arterial blood pressure are also mixed, with four reports of decreased blood pressure, seven studies reporting no change, and two reporting an increase in blood pressure.

To our knowledge, this is the first study to use LDF to measure changes in cerebral perfusion and also the first to report the associated changes in $\mathrm{tPO}_{2}$ during the administration of surfactant. LDF methodology provides a continuous measurement of changes in brain blood flow that correlate well with flow measurements using microsphere $(5,9)$. LDF is capable of measuring rapid changes in CBF associated with acute hypoxia (5) or umbilical cord occlusion (10), so the likelihood that significant changes in CBF-escaped detection in the current experiments are small.

Baseline $\mathrm{tPO}_{2}$ values $(\sim 37$ torr) were well above those reported previously for the chronically instrumented fetal sheep (10 to 12 torr) $(5,10)$ and were similar to the values reported for adult animals breathing $21 \%$ oxygen (11). Administration of poractant alfa resulted in a rapid increase in $\mathrm{tPO}_{2}$ to supraphysiological levels. This could be caused by an increase in $\mathrm{CBF}$ (although not detected, as discussed above), an increase in arterial oxygen content, and a decrease in cerebral oxygen consumption. Although no measurements of cerebral oxygen consumption were made in these experiments, EEG depression after the administration of animalderived surfactants has been reported (12) and would be consistent with decreased cerebral metabolic rate. Whether such rapid increases in cerebral $\mathrm{Po}_{2}$ are of overall benefit is questionable because they do not mimic the parturition of term infants and may expose the preterm infant to elevated levels of reactive oxygen species.

As mentioned above, a number of studies have observed significant decreases in arterial blood pressure in preterm infants after surfactant instillation. The impact of such hypotension on $\mathrm{CBF}$ is of concern in the preterm infant, where autoregulation of CBF may be inadequate (13). However, the current studies demonstrate that, in this preterm lamb model, $\mathrm{CBF}$ is autoregulated against decreases in arterial blood pressure as low as $40 \mathrm{~mm} \mathrm{Hg}$ (Fig. 6). Although the current experiments are the first to demonstrate $\mathrm{CBF}$ autoregulation in an ex utero preterm lamb, the findings are also in accordance with studies of fetal sheep in utero as early as $110 \mathrm{~d}$ gestation (9). However, it is possible that CBF autoregulation is impaired in the infant relative to the preterm lamb and thus caution is warranted in extrapolating lamb studies to the infant.

Surfactant distribution. One goal of surfactant therapy is to achieve an even distribution of surfactant throughout the lung so that alveolar expansion is uniform. Nonuniform alveolar expansion may lead to volutrauma and barotrauma in the overinflated alveoli and underutilization of collapsed alveoli (14). These disturbances may contribute to disruption of lung tissue architecture and chronic lung disease. Variables reported to influence surfactant distribution into the airways include inherent properties of surfactants, such as viscosity, gravity by positioning of the infant, volume of surfactant administered, rate of administration, ventilation settings and the use of PEEP, and fluid volume within the lung (4). The results of this study are in accordance with reports that uniformity of surfactant distribution improves as volume of surfactant administered is increased (3). Optimal distribution can be achieved if a rapidly injected bolus accumulates in the airway to form an occluding column of liquid that is advanced into more distal airways by positive airway pressure (15). At airway branches, the surfactant column is then distributed more evenly between branches, resulting in relatively even distribution relative to alveolar volume. Disruption of the occluding column of surfactant, such that inspiratory air is able to flow past the column, results in suboptimal distribution. Although the occluding column of surfactant was not quantified in this study, it seems likely to have persisted longer with administration of lucinactant given the greater volume administered and comparable viscosity compared with poractant alfa (Nutt et al., Comparison of the novel lung surfactant surfaxin (lucinactant) with currently available commercial products, 2004 Pediatric Research 55:514A). It should also be noted that differences in viscosity and chemical composition might also have played a role, and these were not examined in these experiments.

A possible limitation of this study is that the microspheres may not have been distributed in direct proportion to the surfactants. However, previous studies have demonstrated a close correlation between distribution of microspheres and radioisotope-tagged surfactant $(6,16)$. The microspheres of 1 to $2 \mu \mathrm{m}$ diameter, as used in this study, have been shown by electron microscopy to distribute to the level of the alveolus (6).

In summary, increase in volume of surfactant administered from $2.5 \mathrm{~mL} / \mathrm{kg}$ to $5.8 \mathrm{~mL} / \mathrm{kg}$ was not associated with an increase in peridosing adverse effects in this preterm animal model of respiratory distress syndrome. The surfactant with the greater volume of instillation was associated with more uniform distribution of the surfactant throughout the lungs. 
Although this difference in distribution may not acutely affect gas exchange, additional studies are needed to determine whether nonhomogenous distribution is associated with regional differences in lung expansion that may translate to abnormal lung development and chronic lung disease.

Acknowledgment. We thank Shannon Bragg for her expert technical assistance.

\section{REFERENCES}

1. Bassan H, Feldman HA, Limperopoulos C, Benson CB, Ringer SA, Veracruz E, Soul JS, Volpe JJ, du Plessis AJ 2006 Periventricular hemorrhagic infarction: risk factors and neonatal outcome. Pediatr Neurol 35:85-92

2. Jobe A, Ikegami M, Jacobs H, Jones S 1984 Surfactant and pulmonary blood flow distributions following treatment of premature lambs with natural surfactant. J Clin Invest 73:848-856

3. Gilliard N, Richman PM, Merritt TA, Spragg RG 1990 Effect of volume and dose on the pulmonary distribution of exogenous surfactant administered to normal rabbits or to rabbits with oleic acid lung injury. Am Rev Respir Dis 141:743-747

4. Espinosa FF, Kamm RD 1999 Bolus dispersal through the lungs in surfactant replacement therapy. J Appl Physiol 86:391-410

5. Bishai JM, Blood AB, Hunter CJ, Longo LD, Power GG 2003 Fetal lamb cerebral blood flow (CBF) and oxygen tensions during hypoxia: a comparison of laser Doppler and microsphere measurements of CBF. J Physiol 546:869-878
6. Merritt TA, Kheiter A, Cochrane CG 1995 Positive end-expiratory pressure during KL4 surfactant instillation enhances intrapulmonary distribution in a simian model of respiratory distress syndrome. Pediatr Res 38:211-217

7. Buckberg GD, Luck JC, Payne DB, Hoffman JI, Archie JP, Fixler DE 1971 Some sources of error in measuring regional blood flow with radioactive microspheres. J Appl Physiol 31:598-604

8. Mazela J, Merritt TA, Finer NN 2007 Aerosolized surfactants. Curr Opin Pediatr 19:155-162

9. Muller T, Lohle M, Schubert H, Bauer R, Wicher C, Antonow-Schlorke I, Sliwka U, Nathanielsz PW, Schwab M 2002 Developmental changes in cerebral autoregulatory capacity in the fetal sheep parietal cortex. J Physiol 539:957-967

10. Hunter CJ, Blood AB, Power GG 2003 Cerebral metabolism during cord occlusion and hypoxia in the fetal sheep: a novel method of continuous measurement based on heat production. J Physiol 552:241-251

11. Tsai AG, Johnson PC, Intaglietta M 2003 Oxygen gradients in the microcirculation. Physiol Rev 83:933-963

12. van den Berg E, Lemmers PM, Toet MC, Klaessens J, van Bel F 2010 Effect of the "InSurE" procedure on cerebral oxygenation and electrical brain activity of the preterm infant. Arch Dis Child Fetal Neonatal Ed 95:F43-F58

13. Soul JS, Hammer PE, Tsuji M, Saul JP, Bassan H, Limperopoulos C, Disalvo DN Moore M, Akins P, Ringer S, Volpe JJ, Trachtenberg F, du Plessis AJ 2007 Fluctuating pressure-passivity is common in the cerebral circulation of sick premature infants. Pediatr Res 61:467-473

14. Lewis JF, Ikegami M, Jobe AH, Absolom D 1993 Physiologic responses and distribution of aerosolized surfactant (Survanta) in a nonuniform pattern of lung injury. Am Rev Respir Dis 147:1364-1370

15. Espinosa FF, Kamm RD 1998 Meniscus formation during tracheal instillation of surfactant. J Appl Physiol 85:266-272

16. Ueda T, Ikegami M, Rider ED, Jobe AH 1994 Distribution of surfactant and ventilation in surfactant-treated preterm lambs. J Appl Physiol 76:45-55 